\title{
SOME PSYCHOPATHOLOGICAL PECULIARITIES AT EARLY AND LATE ONSET IN PATIENTS WITH ALZHEIMER'S DISEASE
}

\author{
M. Arnaudova-Jekova \\ Third Psychiatric Clinic, MHAT "St. Marina" \\ Department of Psychiatry, \\ Medical University - Varna, Bulgaria
}

\section{SUMMARY:}

Age-related conditions are of great medical and social importance. The most common cause of dementia is Alzheimer's disease (AD). Neuropsychiatric symptoms are an integral part of the disease and present severe problems to patients, their families, caregivers and the society at large.

The aim of our work was to explore the manifestation of some psychic (non-psychotic and psychotic) and behavioral symptoms (BPSD) of hospitalized patients with early onset (EO-AD) and late onset (LO-AD) of AD with a view to compare and enrich the clinical, diagnostic and differential diagnostic possibilities. In this study significant differences in terms of BPSD between EO-AD and LO-AD patients were found. The data of EO-AD patients to be more psychotic with more behavioral problems could represent another clinical support of dividing AD not only to the age of onset.

As we discuss in our paper some methodological issues, the number of examined patients in our study was comparatively small. That is why we comment some of the data as a tendency.

Key words: early onset Alzheimer's disease (EO-AD), late onset Alzheimer's disease (LO-AD), BPSD, in-patients

\section{INTRODUCTION:}

Alzheimer's disease (AD) is the most common cause of dementia. There is more research data concerning cognitive decline, while some aspects of psychic /nonpsychotic and psychotic/ and behavioral symptoms (BPSD) still remain unstudied. Early onset of Alzheimer's disease (EO-AD) is defined as the disease process that becomes apparent in individuals under about 65 years of age. When comparing EO-AD with late onset (LO-AD) some differences in clinical features were registered by different authors. They put an accent on rapid progression (17), more severe language problems (16), visuospatial dysfunction (13) in EO-AD patients.

BPSD are an integral part of the disease and present severe problems to patients, their families, caregivers and the society at large. The importance of BPSD has become a focus of more special attention only in the 1980s. Later on, in 1999, a definition of the BPSD was accepted (9). Still there are few reports on the differences of BPSD between EO-AD and LO-AD.

The aim of our work was to explore the manifestation of some BPSD in EO-AD and LO-AD with a view to compare and enrich the clinical, diagnostic and differential diagnostic possibilities. This should be a basic prerequisite for optimizing the therapeutic approaches and medico-social care of AD patients.

\section{SUBJECTIVES AND METHODS:}

We examined 38 EO-AD (25 females; 13 males; mean age $60.73 \pm 2.24$ ) and $72 \mathrm{LO}-\mathrm{AD}$ (45 females; 27 males; mean age $75.31 \pm 2.46$ ), hospitalized for the first time at gerontopsychiatric department of University hospital "St. Marina"-Varna in the period 2000-2009.

Clinical examination, The Mini-Mental State Examination (MMSE; Folstein et al., 1975) and Neuropsychiatric Inventory (NPI; Cummings et al., 1994) were used. Patients were diagnosed according to ICD-10, DSM-IV and NINCDS/ADRD criteria for probable AD. Patients were with mild, moderate and severe dementia.

Age of onset was defined as the age when the first symptoms that caused impairment of family, occupational or social functioning appeared. Duration was defined as the period between the onset and the first medical assessment (in our case it was their first hospitalization). The boundary between early and late onset was 65 years old.

\section{RESULTS:}

A variety of psychic /non-psychotic and psychotic/ symptoms were present along with that of dementia in $\mathrm{AD}$ patients. As it is shown on Fig. 1 we found out significantly greater frequency of delusions of referrence and poisoning $(p<0,05)$ and relatively more frequent visual and verbal hallucinations, delusions of harm and symptom of Capgras in $\mathrm{EO}-\mathrm{AD}(\mathrm{p}>0,05)$, while anxiety was significantly greater 
in $\mathrm{LO}-\mathrm{AD}(\mathrm{p}<0,05)$.

On a syndrome (Fig.2) we found out in EO-AD psychotic syndromes (paranoid/paranoid-hallucinatory)$71.05 \%$, anxiety- $47.36 \%$ and depression- $28.94 \%$; in LO-AD patients anxiety $-72.22 \%$, psychotic syndromes- $43.05 \%$, depression- $25 \%$. A comparison on a syndrome level revealed that EO-AD patients were more psychotic $(p<0,05)$, while LO-AD- more anxious $(p<0,05)$.

Confrontation of the psychotic and non-psychotic symptoms in EO-AD and LO-AD with the degree of dementia revealed more frequency of paranoid-hallucinatory syndromes in moderate dementia in EO-AD (Fig.3). Depression in EO-AD and mild dementia (57.74\%) tend to prevail over these in LO-AD (45.83\%). Anxiety in LO-AD patients and mild dementia was significantly greater than in EO-AD patients $(\mathrm{p}<0,05)$.

As a whole, behavioural disorders were more frequent in EO-AD than in LO-AD patients. Summarized, apathy, physically non-aggressive, verbal aggressive and physically aggressive behaviors were significantly more often met in EO-AD patients ( $\mathrm{p}<0,05)$ (Fig. 4).

There was a tendency, behavioral disorders to be rarer in mild dementia and more frequent in moderate to severe dementia in both groups (Fig. 5).

\section{DISCUSSION:}

Before discussing the results we would like to make some reservation. At first, multiple symptoms occurred simultaneously. Second, the sample consisted of in-patients in acute ward. Third, classifying the patients into two groups according to the age of onset was based on caregiver's information. According to some authors current neuropsychiatric measures have some limitations (e.g. acquisition of data is limited to informants or caregivers as respondents, limited depth of items specific to moderate dementia) $(6,24,25)$.

All patients entered the hospital due to some psychotic or behavioral problem and it was their first assessment. Psychotic states presented as paranoid or paranoid-hallucinatory syndromes. Delusions of theft were mostly connected with the memory problems. Psychotic symptoms like delusions and hallucinations provoked agitation and aggression which in most of the cases had the character of defense. Our experience indicates that the initial diagnosis in such cases could be quite difficult in psychotic AD patients in terms of schizophrenia. It is even more difficult in EO-AD. In our practice we use operational criteria for psychosis in AD (20). The diagnostic difficulties could be due also to the fact that usually multiple neuropsychiatry symptoms exist. Some of the symptoms overlap or are difficult to be differentiated from these of dementia or are attributed to the process of aging or even of a somatic problem. The most differential diagnostic difficulties arouse when depression was present in EO-AD with mild dementia during their first hospitalization. On other hand, the unrecognized and untreated depression in old age is associated with progressive disability (14) and increases the risk of developing Alzheimer's disease (19). We did not have patients with depression and severe dementia in either group. The same representation was observed with delusional symptoms. This could be explained with the heavy intellectual and personality decline in severe dementia, which makes nearly impossible the formation and verbalization of psychotic and non-psychotic symptoms. Janzing et al. (2005) described depressive syndromes in patients with mild dementia and proposed that sharing of depressive symptoms reflects relatively intact cognitive functions (18). In our study the comparison on a syndrome level revealed that EO-AD patients were more psychotic, while LO-AD- more anxious.

Behavioral disorders were met at every stage of the illness but with a tendency to prevail in moderate to severe dementia and again in EO-AD patients. Our results confirm the data from other authors that there is some unspecific kind of connection between the growing psychopathology and the cognitive decline $(3,11,21,22,23)$. Behavioural disorders were more frequent in EO-AD than in LO-AD patients. Other authors have found in EO-AD relatively fewer $\operatorname{BPSD}(1,7,15,25)$. We would like to emphasize, that the number of examined patients in our study was comparatively small. That is why we comment some of the data as a tendency. Nevertheless, the data of EO-AD patients to be more psychotic with more behavioral problems could represent another clinical support of dividing AD not only to the age of onset.

\section{CONCLUSION:}

Accurately eliciting BPSD phenomena can be difficult in people with dementia, while the information is derived mainly from interviews with informants, who may over-rate or underestimate some symptoms. Applying clinical examination and observation gives the opportunity of a more precise evaluation of the symptoms.

In this study significant differences in terms of BPSD between EO-AD and LO-AD patients were found. As we mentioned above, the number of examined patients in our study was comparatively small. That is why we comment some of the data as a tendency. Nevertheless, the data of EO-AD patients to be more psychotic with more behavioral problems could represent another clinical support of dividing $\mathrm{AD}$ not only to the age of onset. 


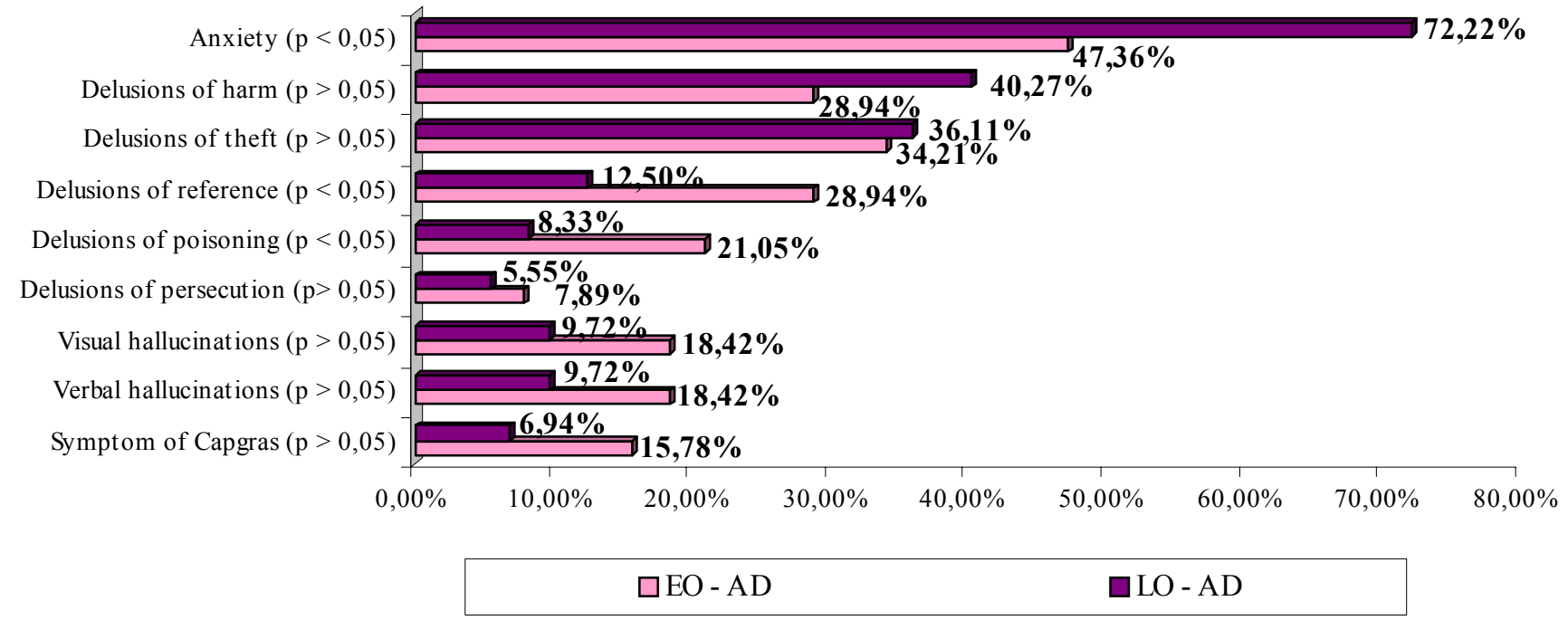

Figure 1. Type and frequency of some psychic (non-psychotic and psychotic) symptoms in EO-AD and LO-AD

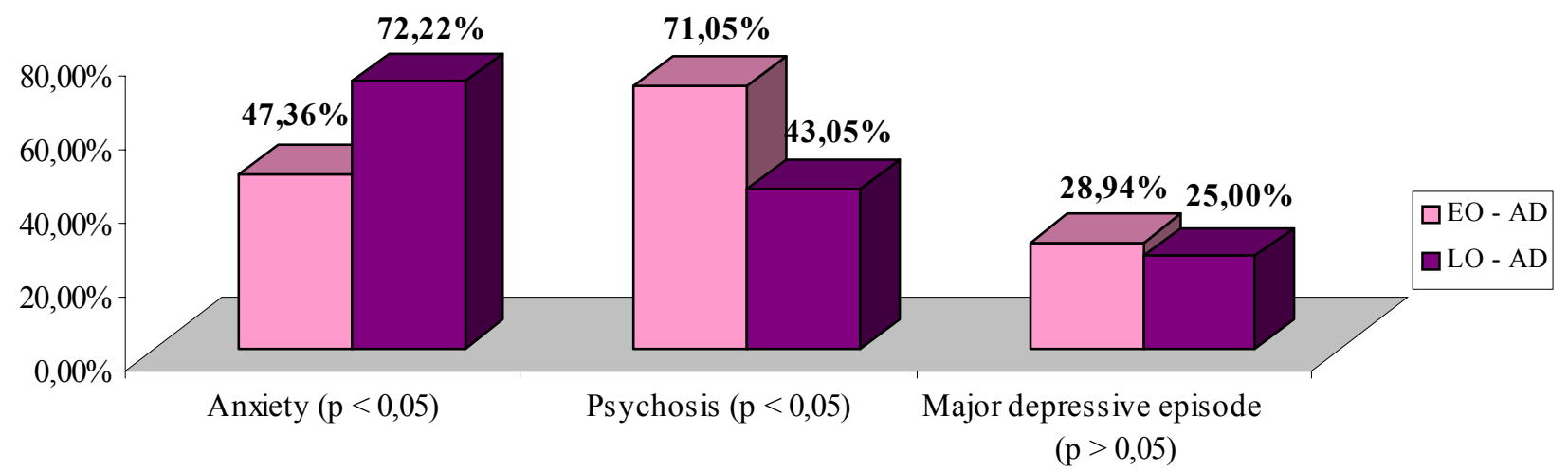

Figure 2. Summarized psychic symptoms in EO-AD and LO-AD

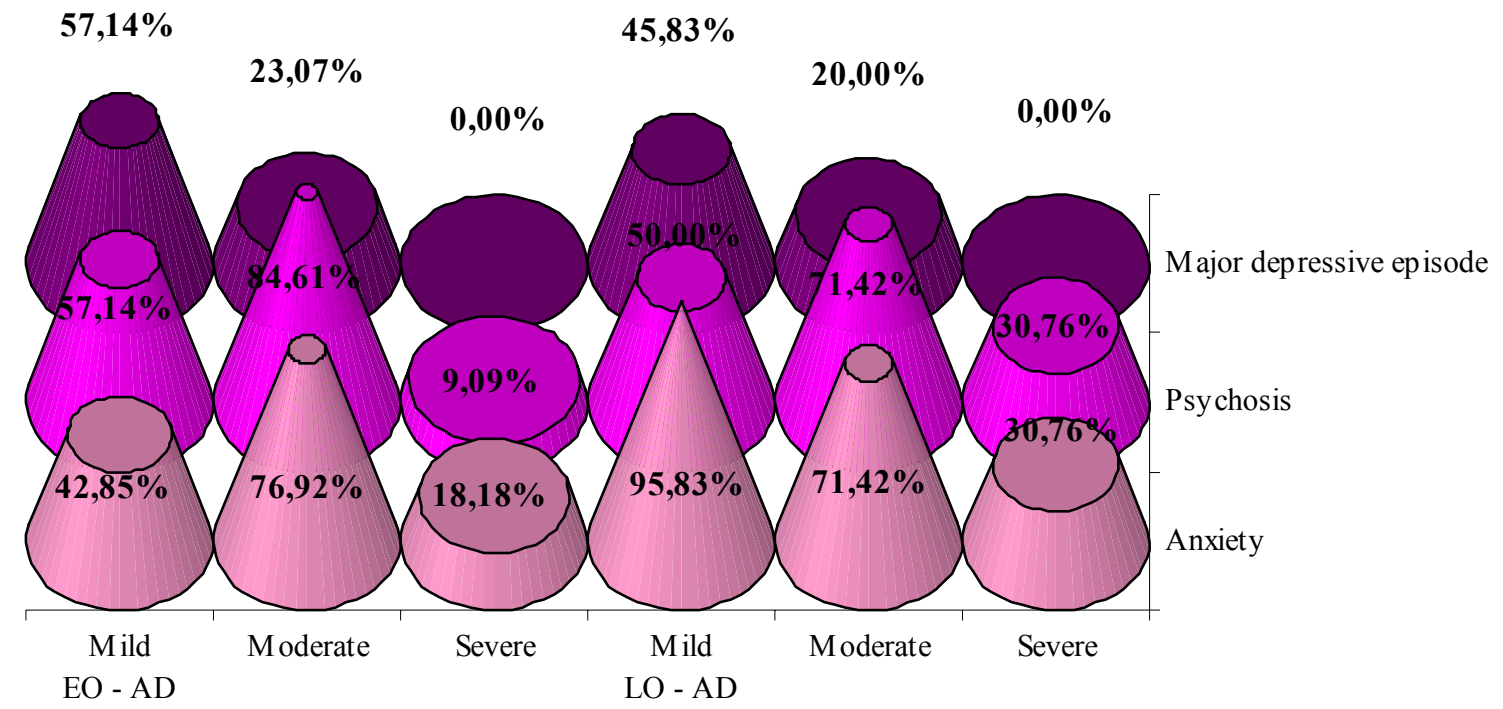

Figure 3. Psychic symptoms in EO-AD and LO-AD and a degree of dementia 


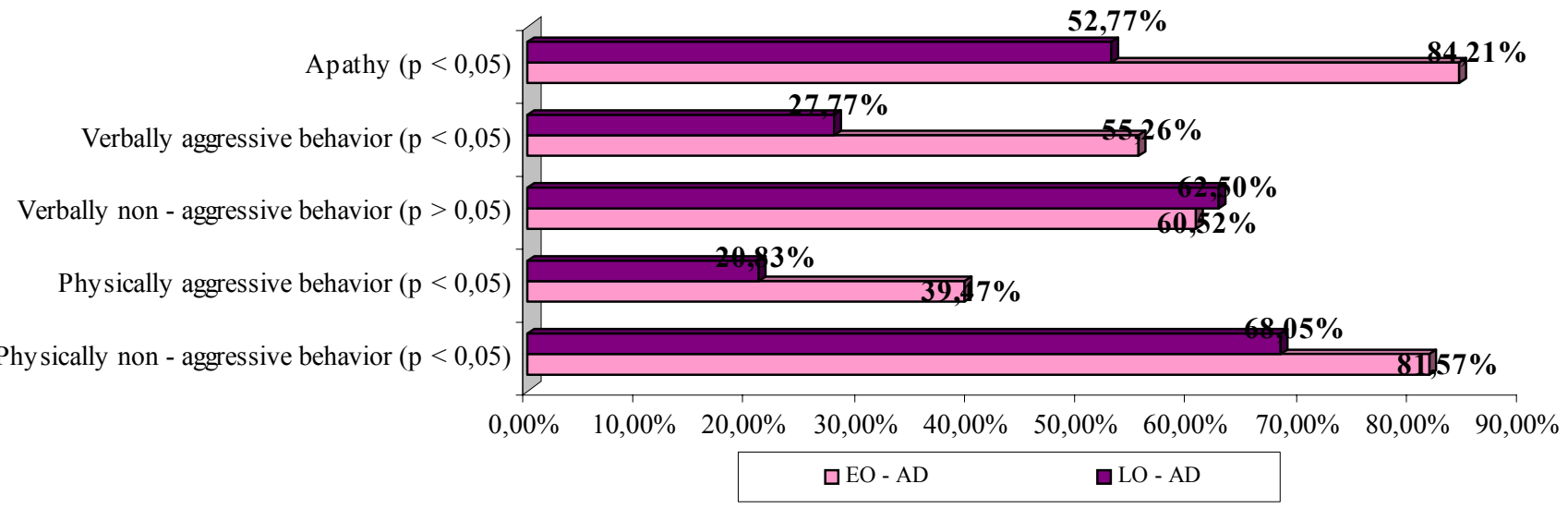

Figure 4. Comparison of the behavioral disorders in EO-AD and LO-AD

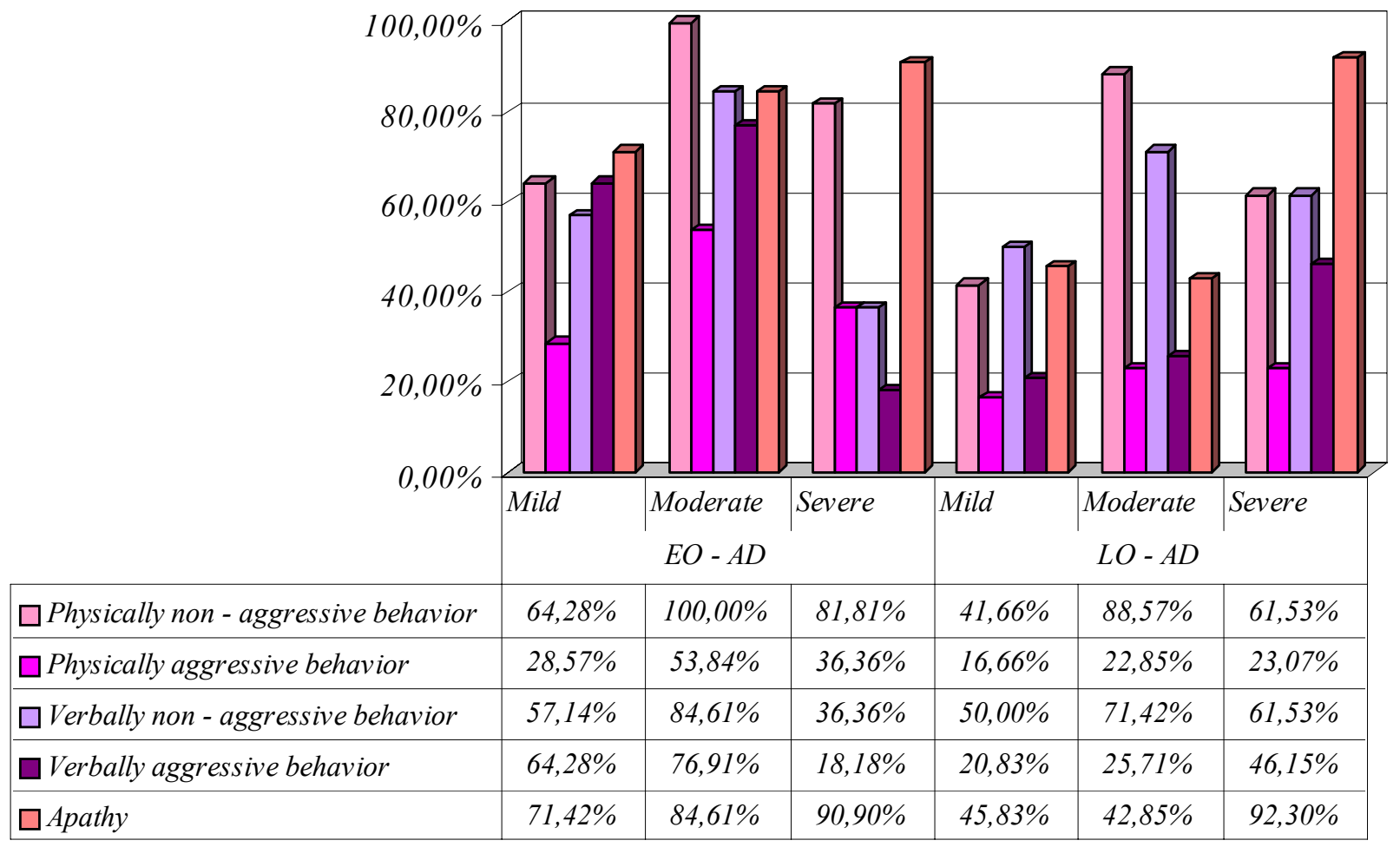

Figure 5. Behavioral disorders in EO-AD and LO-AD and a degree of dementia 


\section{REFERENCES:}

1. Ballard C, Oyebode F. Psychotic symptoms in patients with dementia. Int J Geriatr Psychiatry 1995; 10: 743-752.

2. Balestreri L, Grossberg A, Grossberg GT. Behavioral and Psychological Symptoms of Dementia as a Risk factor for Nursing Home Placement. International Psychogeriatrics 2000; 12(Supll.S1):59-62.

3. Bylsma FW, Folstein M, Devanand DP et al. Delusions and patterns of cognitive impairment in Alzheimer's disease. Neuropsychiatry Neuropsychol Behav Neurol 1994; 7: 98-103.

4. Chemerinski E, Petracca G, Teson A. Sabe L, Leiguarda R, Starkstein SE. Prevalence and correlates of aggressive behavior in Alzheimer's disease. $J$ Neuro-psychiatry Clin Neurosci Nov. 1998; 10:421-5.

5. Cummings JL, Mega MS, Gray K, Rosenberg-Thompson S, Carusi DA, Gornbein J.. The Neuropsychiatric Inventory: Comprehensive assessment of psycho-pathology in dementia. Neurology 1994; Dec;44(12): 2308-2314.

6. de Medeiros K, Robert P, Gauthier S, Stella F, Politis A, Leoutsakos J, et al. The Neuropsychiatric Inventory-Clinician rating scale (NPI-C): reliability and validity of a revised assessment of neuropsychiatric symptoms in dementia. Int Psychogeriatr. 2010 Sep;22(6):984-94. Epub 2010 Jul 1.

7. Ferran J, Wilson K, Doran M et al. The early onset dementias: a study of clinical characteristics and service use. Int J Geriatr Psychiatry 1996; 11: 863-869.

8. Finkel S. Behavioral and psychological signs and symptoms of dementia: Implications for research and treatment. Int Psychogeriatr 1996; (Suppl. 3): 215-552.
9. Finkel S, Burns A. Behavioral and Psychological symptoms of Dementia (BPSD): A Clinical and Research Update. Introduction. Int Psychogeriatr 2000; 12 (Suppl. 1): 9-12.

10. Finkel S, Burns A, Cohen G. Behavioral and Psychological symptoms of Dementia (BPSD): A Clinical and Research Update. Overview. Int Psychogeriatr 2000; 12(Suppl. 1):13-18.

11. Flynn FG, Cummings JL, Gornbein J. Delusions in dementia syndromes: investigation of behavioral and neuropsychological correlates. $J$ Neuro-psychiatry Clin Neurosci 1991; 3(4): 364-70.

12. Folstein MR, Folstein SE, McHugh PR. "Mini-mental state": A practical method for grading the cognitive state of patients for the clinician. $J$ Psychiatr Res 1975; Nov;12(3):189-198.

13. Fujimory M, Immamura $T$, Yamashita H, et al. Age at onset and visuocognitive disturbances in Alzheimer's disease. Alzheimer Dis Assoc Disord 1998; Sep;12(3):163-166.

14. Hargrave R, Reed B, Mungas D. Depressive syndromes and functional disability in dementia. J Geriatr Psychiatry Neurol 2000; 13: 72-7.

15. Holtzer R, Tang MX, Devanand DP et al. Psychopathological features in Alzheimer's disease: course and relationship with cognitive status. $\mathrm{J} \mathrm{Am}$ Geriatr Soc 2003; 51: 953-60.

16. Imamura T, Takatsuki Y, Fujimory $\mathrm{M}$, et al. Age at onset and language disturbances in Alzheimer's disease. Neuropsychologia 1998; Sep;36(9):945949.

17. Jacobs D, Sano M, Marder K, et al. Age at onset of Alzheimer's disease: relation to pattern of cognitive dysfunction and rate of decline. Neurology 1994; Jul; 44(7):1215-1220.

18. Janzing J, Naarding P, Eling $P$. Depressive Symptoms Predict Slow Cognitive Decline in Mild Dementia. Dementia Geriatr Cogn Disord 2005;20:(2-3)77-81

19. Jeffry S. Depression Doubles Risk for Dementia and Alzheimer's disease. Neurology 2010; 75: 35-41, 27-34, 21-26, 12-14.

20. Jeste D, Finkel, S. Psychosis of Alzheimer's disease and related dementia. Am J Geriatr Psychiatry 2000; 8: 29-34.

21. Jeste DV, Wragg RE, Salmon DP, Harris MJ, Thal LJ. Cognitive deficits of patients with Alzheimer's disease with and without delusions. Am J Psychiatry 1992; Feb;149(2):184-9.

22. Lawlor BA, Swanwick G, Coen R. Relationship Between Cognition and Behavioral and Psychological Symptoms of Dementia. International Psychogeriatrics 2000; 12 /Suppl.S1/:165-170.

23. Migliorelli R, Petracca G, Teson A, Sabe L, Leiguarda R, Starkstein SE. Neuropsychiatric and neuropsycho-logical correlates of delusions in Alzheimer's disease. Psychol Med 1995; May; 25(3): 505-13.

24. Oppenheim G. The earliest signs of Alzheimer's disease. J Geriatr Psychiatry Neurol 1994; 7:116-120.

25. Toyota Y, Ikeda M, Shinagawa S, Matsumoto T, Matsumoto N, Hokoishi K. Comparison of behavioral and psychological symptoms in early-onset and lateonset Alzheimer's disease. Int J Geriatr Psychiatry 2007; Sep;22(9):896-901.

\author{
Address for correspondence: \\ Mariana Arnaudova-Jekova, MD, PhD \\ Third Psychiatric Clinic MHAT "St. Marina" \\ 1, Hristo Smirnenski str, 9010 Varna, Bulgaria \\ mobile: $+359 / 888512627$ \\ e-mail:marnaudova@hotmail.com
}

